\title{
Index to genera and species, Volume 11 (2000)
}

$\mathbf{A}$

Abies 208, 234-235

- alba 231-235

- grandis 185

- sibirica Ledeb. 59, 61

Abrolophus Berlese 191-193

- neobrevicollis Zhang \& Goldarazena 192

- brevicollis (Oudemans) 187, 191

- fuliginosum 192

Absinthium 204

Acer 142

- platanoides 232

Acherontia atropos (L.) 135

Achillides cf. maackii Men. 152

Acipenser stellatus Pallas 9

Acleris emargana (F.) 134

- laterana (F.) 134

- notana (Don.) 134

Acrolepiopsis assectella (Zell.) 133

Acronicta lutea Brem. \& Grey 154

- major atritaigensa Dubat. \& Zolot. 152, 155

- major Brem. 154-155, 157

- major major Brem. 152, 155

Actias Leach 153

- gnoma Btl. 155

Adelges piceae (Ratzeburg) 208

Adelurola Strand 239

- florimela Foerster 239

- florimela (Haliday) 239-242

Aegopodium podagraria L. 49

Aelurillus festivus 70

- v-insignitus (Clerck) 70

Aesculus hippocastanum L. 52

Aglais 152

Aglaostigma 88

Aglia tau L. 152

Agonopterix heracliana (L.) 135

Agriphila selasella (Hbn.) 134

Agrius Hbn. 153

Agrochola helvola (L.) 135

Ahlbergia frivaldszkyi Led. 154-155
Allothrombium Berlese 187, 190, 192

- fuliginosum (Hermann) 187, 190

Alnus 52, 133, 142, 223

- alba 235

- glutinosa (L.) 142

- hirsuta (Spach.) 143

- incana (L.) 142

Alysia (Alysia) florimela Haliday 239

- (Alysia) perdita Haliday 241

- striolata Thomson 242

Amphipoea 56

- crinanensis 56

- fucosa (Frr.) 135

Amurilla subpurpurea Btl. 155

Ancylis apicella (Den. \& Schiff.) 134

- mitterbacheriana D. \& S. 154

- nemorana Kuzn. 154

Andricus kollari 48

Angelica 203

- sylvestris 203

Anisocyrta Foerster 239-240

- alpinicola van Achterberg 239-240

- longicauda Tobias 241

— perdita (Haliday) 239, 241

Anobium emarginatum 52

- rufipes Fabricius 49, 52

Antheraea Hbn. 153

Anthonomus varians 62

Anthriscus sylvestris (L.) Hoffm. 45, 53

Antichaeta atriseta (Loew) 3

Apamea furva (Den. \& Schiff.) 135

- unanimis 56

Apatura F, 151, 153, 155, 157

- ilia D.\&S. 151

- iris L. 151, 154

- metis Frr. 151

Apion opeticum Bach 246

Apis cerana 194

- dorsata 194

- florea 194

- mellifera 194

Apoda Haw. (=Cochlidion Hbn.) 153 
Aporia crataegi (L.) 134

- hippia Brem. 155

Apotomis 136

- fraterculana Krogerus 131, 134

Arabidopsis 114, 186

Araeopteron Hmps. 153

Arge 88

Argyresthia goedartella (L.) 133

Argyronome laodice Pall. 151, 153

Arichanna melanaria (L.) 135

Artemisia 7-8, 234

- austriaca 7, 9-10, 13, 18, 20

Asalebria pseudoflorella (A. Schmidt) 9

Asianellus festivus (C.L. Koch) 70

Aspistes Meigen 119

- antydactylatis Cook 122

- berolinensis Meigen 119-121

- freyi Cook 119, 121-122

- helleni sp.n. 119-120, 122-123

Astrenis Förster 195, 198, 200, 208, 223, 225

- brunneofacies Vikberg 195, 198, 199, 200 , 209, 213, 225

- densa Chiu in Chiu \& Wong 208

- nigrifacies Vikberg sp. n. 198, 209-210

- paradoxus (Schmiedeknecht) 198

- sinuatus (Roman) 198

- granum Chiu 208

- nigrifacies 225

—nigrifacies Vikberg sp. nov. 195, 210, 212, 214

- paradoxus 223

- paradoxus (Schmiedeknecht) 195, 212

- sinuatus (Roman) 200, 208

Astrenus 223

Asyntactus Marshall 239, 242

- rhogaleus Marshall 239,241-242

- sigalphoides Marshall 242

Atalophlebia 95

Attus arenicolor Grube 80

- castriesiana Grube 80

- flavoater Grube 73

- fusconotatus Grube 72

- melanotarsus Grube 70

- nobilis Grube 78

- striatipes Grube 85

Autographa gamma (L.) 135

- jota 56
B

Balaustioides tuxeni Southcott 187, 191-192

Balsa Wlk. 153

Belenois crawshayi Btl. 152

Bellardia stricta (Villeneuve) 3

Belothrips 191

Bergenia crassifolia (L.) Fritsch. 61

Beris fuscipes Meigen 2

- strobli Dusek \& Rozkosny 2

Betula 7, 142, 196, 206, 211, 223

- alba L. 142

Bianor aemulus (Gertsch) 71

- aenescens Dunin 71

- aurocinctus (Ohlert) 71

— aurocinctus Logunov \& Marusik 71, 75

Biorrhiza pallida (Olivier) 51

Biselachista kebneella (Traugott-Olsen \& Schmidt-Nielsen) 131, 133, 136

Blastesthia turionella (L.) 134

Bledius defensus Fauvel 230

Bobeckia striolata 242

Boletophagus reticulatus (L.) 191

Bombus 218

- argillaceus 218

- hortorum Mehlbeere 218

- agrorum (Fabricius) 217-218

- lapidarius (Linnaeus) 218

-pascuorum (Scopoli) 218

Boraginobius Wagner 245

Borkhausenia fuscescens (Hw.) 133

Brachonyx pineti 62

Brachygluta haematica (Reichenbach) 230

Brachylomia viminalis (F.) 56, 135

Brachynotocoris puncticornis Reuter 237

Brachypodium pinnatum (L.) 58

Bracon 125

- (Lucobracon) erraticus Wesmael 126, 127

- (Lucobracon) flagellaris Thomson 126-127

- (Lucobracon) polaris Hellén 125-126

- dallatorrei Szépligeti 127

- erraticus Wesmael 127-128

- flagellaris Thomson 128

- polaris Hellén 125-128

Brassica 186

Butomus umbellatus 243 
C

Cacile maritima 4

Callicorixa praeusta (Fieber) 184

Callimorpha dominula L. 156

Calliphora subalpina (Ringdahl) 3

Callistege mi (Cl.) 135

Caloptilia elongella (L.) 133

Camptobrochis Fieber 233

- (K.) putoni 234

Camptobrochis punctulatus Fallén 233

Caragana 7

Carex 58, 230

Carpinus 142, 144

Carrhotus detritus 71

- xanthogramma (Latreille) 71

Catocala adultera Mén. 135

— dula Brem. 154, 157

- sponsa L. 154

Cedestis farinatella (Dup.) 133

Cedrus 237

Celaena haworthi (Curt.) 135

Celia curruca Dahlbom 36-37

— troglodytes (Lind.) 35

Celtis 144

Celypha rufana (Sc.) 134

Cerastis rubricosa (Den. \& Schiff.) 135

Ceutorhyncellus cakilis (Hansen) 4

Ceutorhynchus Germar 245

- larvatus Schultze 245-246

- pallidicornis Gougelet \& Brisout de Barneville 230, 245, 256

Chaetostevenia brevicornis 1

Chalcoscirtus (Chalcosibiricus) alpicola (L. Koch) 71

- (Chalcosibiricus) glacialis Caporacc 72

- (Chalcosibiricus) hyperboreus Marusi 72

- (Chalcosibiricus) tanyae Logunov \& M 72

- alpicola 71

- carbonarius Emerton 71

- glacialis 68,72

- glacialis sibiricus Marusik 72

- grishkanae Marusik 72

- hyperboreus Marusik 72

- micans (Caporiacco) 68

-tanyae Logunov \& Marusik 72

Charletonia Oudemans 191-193

- cardinalis (C.L. Koch) 187, 191

- singularis (Oudemans) 191-192
Cheilosia sootryeni 1

- vernalis (Fallén) 1

Chersotis cuprea (Den. \& Schiff.) 135

Chionodes electella (Zell.) 134

Chloroclysta v-ata Hw. 151

Chlosyne Butler 167, 173

- janais 174

- lacinia 174

Chrysoesthia sexguttella (Thnbg.) 134

Chytonix Grt. 153

Cicadetta montana Scopoli 190

Clambus Fischer von Waldheim 230

- gibbulus (Le Conte) 230

- nigrellus Reitter 229-230

- puctulum (Beck) 230

Clethrionomys glareolus Schreb. 191

Cloeon 89-90, 94

Cochylis nana (Hw.) 134

Coenocalpe lapidata (Hbn.) 134

Coenonympha leander Esp. 151, 156

- sarcania L. 151, 156

Coleophora milvipennis Zell. 133

- vestianella (L.) 134

Condica Wlk. 153

Corixa dentipes (Thomson) 184

Corylus 233

- avellana 194

Cotesia bignellii (Marshall) 172

- margiventris 185

— melitaearum (Wilkinson) 169

Creophilus erythrocephalus (Meyer-Rochow) 130

Cybosia mesomella 56

Cydia coniferana (Saxesen) 135

- indivisa (Danil.) 134

Cymatia bonsdorffii (C. Sahlberg) 183

\section{D}

Dahlica charlottae (Meier) 133

Damora sagana Dbld. 154

Dapsilarthra (Adelurola) florimela 239

- florimela 239

Demophorus Thomson 215

Dendryphantes atratus 82

— biankii 72

- czekanowskii Prószynski 72

- fusconotatus (Grube) 72

— rudis (Sundevall) 73 
Depressaria sordidatella Tgstr. ( =weirella $\mathrm{Stt}$. 133

Deraeocoris 233-234

- approximatus Stål 236

- flavilinea (Costa) 237

- lutescens 232-233

- (Knightocapsus) lutescens (Schilling) 231233

-(C.) punctulatus 234

- lutescens (Schilling) 231-234, 237

- putoni Montandon 233

Diachrysia (chrysitis) chrysitis 56

- (chrysitis) tutti 56

Dianous coerulescens (Gyllenhal) 229

Diarsia florida 56

- rubi (View.) 135

Dicranura stsvetajevi Schintlm. \& Svir. 154

—ulmi D. \& S. 154

Dioryctria schuetzeella (Fuchs) 134

Dryobius roboris (L.) 190

Dysgonia Hbn. 153

Dyspetes Förster 88

- arrogator Heinrich 88

- fracticeps Townes 88

- praerogator (Thomson) 88

- praerogator Thomson 88

\section{$\mathbf{E}$}

Earobia 195, 206, 208

- minor Townes \& Townes 206-207

- paradoxa (Perkins) 198-199, 206-207, 225

Echthrolaricobius Perkins 206

- paradoxus Perkins 206

Ectoedemia minimella (Zett.) 131

Eilema complanum 56

- lurideolum 56

Ematurga amatoria L. 191

Empusa muscae 129

Entomophtora muscae 130

Ephestia elutella (Hbn.) 134

- kuehniella (Zeller) 134

Epicallia villica L. 151, 156

Epinotia brunnichana (L.) 134

-demarniana (F. v. R.) 134

- maculana (F.) 134

- nisella (Cl.) 134

- solandriana (L.) 134

Epione repandaria (Hufn.) 135
Epiplema H.-S. 153, 155

- fuscifrons Wrr. 155

Epistathmus crassicornis Horstmann 225

Eretmocera medinella (Staudinger) 21

Ergane albifrons Kulczynski 73

Erythraeus Latreille 191-192

- gertrudae Haitlinger 187, 191

- monikae Haitlinger 187, 191

Eucosma guentheri (Tgstr.) 134

- hohenwartiana (Den. \& Schiff.) 134

Eudocima Billb. 153

Euleia cognata (Wiedemann) 240

Euophrys 73

- erratica 70,82

- flavoatra (Grube) 73

- frontalis (Walckenaer) 73

- iwatensis 82

- kataokai 73

— proszynskii Logunov, Cutler \& Marusik 73

Eupelmella vesicularis 51

Euphydryas 167-168, 173-174

- aurinia (Rottemburg) 167-168, 172-174

- maturna (L.) 167-168, 171-174

Eupithecia indigata (Hbn.) 135

Euplagia quadripunctaria Poda 156

Eurodryas 167

Eurrhypara hortulata (L.) 134

Eutelia Hbn. 153

Euxoa decora 56

Evarcha albaria (L. Koch) 73

- arcuata (Clerck) 74

- falcata (Clerck) 68, 74-75

- laetabunda (C.L. Koch) 74

- michailovi 74

- proszynskii Marusik \& Logunov 68, 74

Eversmannia Chr. 155

- exornata (Eversmann) 151, 155, 157

Exaeretia ciniflonella (Zell.) 133

Exapate congelatella (Cl.) 134

Exoteleia dodecella 134

$\mathbf{F}$

Fagus 142, 144

Fixsenia herzi Fixs. 155

Fomes fomentarius 229

Fragaria 114

Fraxinus 142 


\section{G}

Glaenocorisa 184

- cavifrons (Thomson) 183

- propinqua (Fieber) 183

Gnypeta ripicola (Kiesenwetter) 229-230

Gordius 129

Grandjeanella 193

Graphiphora augur (F.) 135

H

Habrosyne Hbn. 153

- pyritoides Hfn. 151, 153

Hada plebeja (L.) (=nana Hfn.) 136

Hambergiella Roman 208

- sinuata Roman 208, 210

Harmochirus latens (Logunov) 70, 75

— nigriculus Logunov \& Wesolowska 71, 75

Harmochirus pullus (Bösenberg \& Strand) 75

Hauptmannia Oudemans 191-192

- wratislaviensis Haitlinger 187, 191

Heamatopota pluvialis (L.) 191

Helicophagella crassimargo (Pandellé) 3

Heliophanus baicalensis Kulczynski 75

- camtschadalicus Kulczynski 75

-dubius C.L. Koch 76

- flavipes (Hahn) 76

- lineiventris Simon 76

— patagiatus Thorell 76

- ussuricus Kulczynski 75-76

Heliothis maritima 56

- viriplaca 56

Hemaris radians Wlk. 155

Hesperocorixa linnaei (Fieber) 184

Horisme tersata (Den. \& Schiff.) 135

Hydraecia 56

- micacea 135

- nordstroemi 56

- ultima 56

Hylobius 57-59, 61, 63-64

- abietis 57, 59-62, 64

- abietis haroldi (Fang San-Yang) 61

- albosparsus Motschulsky 57, 59-64

- congener Dalla Torre, 64

- gebleri Boheman 60-61, 64

- haroldi Faust 60-61, 64

- piceus De Geer 60-61, 64

- pinastri Gyllenhal 59-63
- transversovittatus Goeze 60-61, 64

Hypenodes Dbld. 153

Hyperaspis 109, 182

- campestris (Herbst) 110, 112

- concolor Suffrian 109-112

- inexpectata Günther 109-112

- pseudopustulata Mulsant 109-111, 182

- reppensis (Herbst) 109, 111-112

Hypocala Gn. 153

Hypodryas 167

\section{I}

Icius 69,81

- abnormis 81

- castriesianus 80

- difficilis 80-81

- nigra Peelle \& Saito 68-69

- parvus 81

- popovi Prószynski 81

Idia $\mathrm{Hbn} .153$

Ilex 144

Iphiclides podalirius L. 156

Ixodes ricinus 124

\section{$\mathbf{J}$}

Juglans 142, 144

- mandshurica Maxim. 154

\section{$\mathbf{K}$}

Kaleva livida Graham 51

Kirinia epimenides Mén. 155

Knightocapsus 233-234

Ktenostilpnus Strobl 215

- aequearticulatus Strobl 215, 217-218

\section{$\mathbf{L}$}

Lagria hirta L. 190

Lampronia fuscatella (Tgstr.) 133

Lamprotes c-aureum 56

Laricobius erichsonii Rosenhauer 207-208

Larix 59, 61, 211

- dahurica Turcz. (= L. gmelinii (Rupr.) 61

- gmelinii (Rupr.) 61

- sibirica 61

Lasiocampa quercus (L.) 135 
Lassenia 192

Lathrolestes 203

Lathyrus vernus 246

Latoia Guer. (=Parasa Moore) 153

Lemonia dumi L. 156

Leptidea sinapis (L.) 135

Leptus 190, 192-193

- beroni Fain (syn. L. holmiae Southcott) 187, 190-191

- echinopus Beron 187, 191

- gabrysi Southcott 190

- holmiae Southcott 190

— ignotus (Oudemans) 187

- laplandicus 187

- mariae Haitlinger 187, 190

- miromiri 192

Leucapamea askoldis Obth. 154

Leucopaxillus giganteus Fr. 241

Lilioceris lilii (Scop.) 4

Limenitis 155

- camilla L. 151, 153, 155, 157

- helmanni duplicata Stgr. 152, 155

- helmanni helmanni Led. 152, 155

- helmanni Led. 154, 155, 157

- sydyi latefasciata Mén. 152, 155

— sydyi Led. 154-155, 157

— sydyi sydyi L. 152, 155

Limnephilus externus Hagen 4

Linosyris villosa (L.) 20

Lithina chlorosata (Scopoli) 191

Lochmaea caprae (L.) 190

Lonchetron fennicum Graham 51

Longitarsus pratensis (Panz.) 4

Lonicera xylosteum 172

Lophopilio (=Odiellus) palpinalis (Herbst) 191

Lucobracon (Fahringer) 125, 127

Luzula (Traugott-Olsen \& Schmidt-Nielsen) 133

Lychnis 114

Lycia hirtaria (Cl.) 135

Lycophotia porphyrea (Den. \& Schiff.) 135

Lyctus africanus Lesne 2

- brunneus Stephens 2

Lygurus 223

Lygus 233

\section{M}

Macroneura vesicularis (Retzius) 51
Maevia castriesiana 80

Magdalis 62, 64

- fermaneki Reitter 63

Magnolia 144

Malacosoma Hbn. 153

- neustereustria L. 153

- neusterium L. 153

- neustrium L. 151

Malus 159, 233

- baccata Borkh. 143

Marbissa dersuuzalai Logunov \& Wesolowska 78

- dybowskii Kulczynski 77

- elongata 78

- koreanica 77

— magister 77, 78

- milleri (Peckham \& Peckham) 77

- nobilis 78

- obscura 77

- pichoni (Schenkel) 69, 77-78

- pomatia (Walckenaer) 69,77

- pulchra 69,78

- pulla (Karsch) 77

— radiata (Grube) 69,77

— zebra Logunov \& Wesolowska 78

Melampyrum 172

- pratense 171-172

Melanargia halimede Mén. 155

Melipona 194

Melitaea 167-168, 173

_-athalia (Rottemburg) 167-168, 170-171, 173

- cinxia (L.), 167-169, 173-174

- diamina (Lang) 167-168, 170, 173-174

Mellicta Billberg 167

- athalia 174

- aurelia Nick. 156

Mendoza canestrinii (Ninni in Canestrini \& Pavesim 69,77

— dersuuzalai (Logunov \& Wesolowska) 78

- elongata (Karsch) 69,78

- nobilis (Grube) 69, 78

- pulchra Prószynski 69

- zebra (Logunov \& Wesolowska) 78

Menemerus pulla 77

Mengersenia Schmiedeknecht 208

- paradoxa Schmiedeknecht 208, 212, 220

- sinuata 210

Mesapamea secalis 56 
Metopia argyrocephala (Meigen) 3

Metopomyza ornata (Meigen) 242

Microbregma emarginata (Duftschmid) 52

Mitopus morio (F.) 191

- ruzikai Silh. 191

Mocis Hbn. 153

Mustela erminea L. 191

Myrica 211

Myrmarachne formicaria (De Geer) 78

- inermichelis Bösenberg \& Strand 69, 79

- japonica (Karsch) 69,78

- lugubris (Kulczynski) 69,79

Mythimna straminea 56

\section{$\mathbf{N}$}

Napomyza elegans (Meigen) 242

Nematopogon magnus (Z.) 135

Neodaruma tamanukii Mtsm. 155

Neon reticulatus (Blackwall) 79

Neorhacodes enslini (Ruschka) 51-52

Neozephyrus japonicus Mrr. 155

Neptis tshetverikovi Kurentz. 155

Nerice Wlk. 153

Netelia Gray 140

— arabs (Strand) 140

- caucasicus (Kokujev) 140

Nicotiana 185

Niditinea 136

- striolella (Matsum.) 133

- truncicolella (Tgstr.) 133

Niphanda fusca Brem. \& Grey 155, 157

Noctua fimbriata 56

- pronuba 56

Nomophila noctuella (Den. \& Schiff.) 134

Nordmannia prunoides Stgr. 154-155

- w-album Knoch 150, 155

Nosema 130

Notonecta 183

- glauca Linnaeus 183

- lutea Müller 183

Notophrudus 223

Nycteola asiatica (Krul.) 4

Nymphula stagnata (Don.) 134

O

Odontomyia argentata (Fabricius) 2
Oligia fasciuncula (Haw.) 4

- leuconephra Hmps. 151

Oligolophus tridens (C.L. Koch) 191

Omiodes Gn. (=Hedylepta Led.) 152

Operophtera brumata (L.) 135

- fagata (Scharfenb.) 135

Oreta Wlk. 153

Orgyia antiqua (L.) 135

Orona petiolaris 208

Orthops 236

— basalis (A. Costa) 233

- rubricatus (Fallén) 236

Orthosia gothica (L.) 135

Otiorhyncllus corruptor (Hast) 2

Oxalis acetosella 114

Oxyethira boreella Svensson \& Tjeder 2, 4

- falcata Morton 2

- falcata Norton 4

\section{$\mathbf{P}$}

Padus avium Miller 142

Pandemis cinnamomeana (Tr.) 134

Pangrapta Hbn. 153

Paniscus arabs Strand 140

- caucasicus Kokujev 140

- nigrithorax Habermehl 140

Paracorixa concinna (Fieber) 184

Paramidea Kusn. 153

Parasarcophaga caerulescens (Zetterstedt) 3

Parascotia cognata Stgr. 154

Parascythris muelleri (Mann) 21

Parasiccia altaica Led. 152, 154-155

Parastichtis suspecta 56

Parnassius mnemosyne L. 151, 154, 156

- stubbendorffii Mén. 154

Paropheltes Cameron 140

Pastinaca sativa 45

Pegomya hyoscyami (Panzer) 240

— nigritarsis (Zetterstedt) 240

— pallidoscutellata (Zetterstedt) 241

Pellenes (Pelmultus) gobiensis Schenkel 79

- (Pelpaucus) ignifrons (Grube) 79

— (Pelpaucus) limbatus Kulczynski 79

_ (s.str.) sibiricus Logunov \& Marusik 70, 80

- (s.str.) tripunctatus (Walckenaer) 70,80

- striatipes 85

Peridea Steph. 153 
Petasites 1

Peucobius 223

Phaestacoenitus 195, 197, 203, 225

- bidentator Aubert 203

- caucasicus Kasparyan 203

- demeyerei Smits van Burgst 203

- niger Kasparyan 203

- niger nitidus Kasparyan 198-199, 203, 225

- caucasicus 225

- rufus Kasparyan 203

- niger nitidus 225

Phalangium opilio (L.) 191

Pheosia tremula (Cl.) 135

Philaeus bicolor 80

- chrysops (Poda) 80

Phintella 69

- abnormis (Bösenberg \& Strand) 70

- arenicolor (Grube) 80

- castriesiana (Grube) 80

- linea (Karsch) 81

- mellotei 80

- parva (Wesolowska) 81

- popovi (Prószynski ) 70, 81

Phlegra fasciata (Hahn) 81

Phlogophora Tr. 153

Phradis Förster 206

Phrudus 198, 200, 208, 215, 223, 225

— angustus 215

- badensis Hilpert 195-196, 199, 221-223, 225

- compressus Vikberg sp. n. 195, 220-201, 225

- dakota Cushman 215

- defectus Stelfox 195, 199, 210, 212, 218, 222, 225

- exarealatus Cushman 215

- femoralis 217

- linorum 215

- longius Chiu in Chiu \& Wong 215, 222

- monilicornis (Bridgman) 199-200, 215, 218,225

- montanus 215

- sinuatus 210, 212

Phyciodes Hübner 167, 174

Phycitodes binaevellus (Hb.) 134

Phyllobius urticae (De Geer) 190

Phyllonorycter 175, 177, 179

- brevilineatella (Benander) 175-176, 179

- corylifoliella (Hubner) 133, 179

— dubitella (Herrich-Schäffer) 175, 179

— grönlieni (M.Hering) 175-176
— heringiella (Grönlien) 175-180

- heringiella f. "aurea" 178-180

- hilarella (Zetterstedt) 175-176

- salictella 175-180

- salictella $f$. viminiella 177-180

- viminiella (Sircom) 175-177, 179-180

Phyllotreta undulata Kutschera 191

Phytocoris lutescens 233

Phytomyza scolopendri Robineau-Desvoidy 243

Picea 59, 61, 206, 212, 218, 220, 223, 225, 235

- abies (= excelsa) 235

- abies (L.) 195-196, 211, 220-221, 223

- abies L. 52, 235

- excelsa 235

- obovata Ledeb. 61

Pierretia sexpunctata (Fabricius) 3

Pimpinella saxifraga 45

Pinalis atomarius 231

Pinalitus Kelton 236

- atomarius (Meyer-Dür) 231, 234-235, 236237

- brachycnemis (Reuter) 237

- rubricatus 236

Pinus 59, 206, 212, 218, 220, 223, 225, 235

- koraiensis Sieb. et Zucc. 61

- obovata 61

_ pumila (Pall.) 61

- sibirica Du Tour 59,61

- sylvestris L. 59, 61, 114, 195-196, 220221, 223, 232

- tabulaeformis Carr. (= P. funebris Komar.) 61

Pissodes 64

- castaneus De Geer 61-63

- pini 62

- piniphilus 62

Plagodis pulveraria (L.) 135

Plantago lanceolata 169,171

Pleurozium schreberi (Brid.) 58

Plexippoides regius Wesolowska 81

Plusiodonta Gn. 153

Podothrombium Berlese 187, 189, 192

- crassicristatum Feider 190

- dariae Haitlinger 189

— kordulae Haitlinger 187, 189-190

- paucisetarum Zhang \& Xin 189-190

- piriformis Robaux 189

- proti Haitlinger 189-190

- rigobertae Haitlinger 190

- roarin. sp. 187-189 
- shellhammeri Robaux 189

- svalbardense Oudemans 189

- sylvicolum Zhang \& Jensen 190

- tersonderi Haitlinger 190

- tymoni Haitlinger 190

Pollenia labialis Robineau-Desvoidy 3

Polychrysia esmeralda 56

- moneta 56

Polygonia c-album (L.) 134

Polyommatus eumedon (Esp.) 135

Pontia 152

Populus 7, 142, 177

- tremula L. 135, 142

- tremula L. x tremuloides Michx. 45

Probles (Rugodiaparsis) crassipes (Thomson) 225

Proserpinus Hbn. 153

Prunus padus 232

Pseudeuophrys erratica (Walckenaer) 70

- iwatensis (Bohdanowicz \& Prószynski) 70, 82

Pseudicius chikunii Logunov \& Marusik 82

- orientalis 82

- vulpes (Grube) 82

Pseudobaptria corylaria Graes. 151

Pseudonaclia puella Bsd. 152

Pseudotsuga menziesii 235

Psyche casta (Pallas) 133

- norvegica (Schöyen) 133

Psylliodes marcida (Ill.) 4

Pterocarya 144

Ptilodon capucina (L.) 135

Pulmonaria 245-246

- obscura 245

Pygmaeolus Hellén 195, 197, 204, 206

— nitidus (Bridgman) 195, 204, 206, 225

- turcicus 204

Pyrgus maculatus Brem. \& Grey 154

\section{Q}

Quercus L. 7, 48, 142, 159, 232-233

- mongolicus Fischer ex Ledeb. 143, 154

- mongolica Fischer 144

- robur L. 49, 51, 144, 194, 232, 234
$\mathbf{R}$

Raphia Hbn. 153

Renocera pallida (Fallén) 3

Rhene atrata (Karsch) 82

Rhipicephalus 124

- oreotragi 124

- sanguineus 124

- warburtoni 124

Rhodiola rosea L. 61

Rhyparioides metelkana Led. 151

Rilaena (=Platybunus) triangularis (Herbst) 191

Rubus 51

—idaeus L. 43, 46, 51, 53

$\mathbf{S}$

Salix 142, 176-177, 179, 196, 234

- alba 177-179

- aurita 177

- caprea 177

- daphnoides 177

- fragilis 177-179

- myrsinifolia 177

- pentandra 177

- phylicifolia 179

- phylicifolia (=bicolor) 177

- purpurea 177

- repens ssp. argentea 177

— siuzewii 177

— viminalis (=rossica) 177

Salticus cingulatus (Panzer) 82

- latidentatus Roewer 82

- lugubris Kulczynski 79

- scenicus (Clerck) 83

Sambucus 46, 51

- racemosa L. 51

Sarcopolia illoba Btl. 152, 154-155

Saturnia Hbn. 153

Schinia nuchalis 56

- scutosa 56

Scleropterus serratus (Germar) 230, 246

Scoliopteryx libatrix (L.) 135

Scolitantides orion Pallas 113-116

Scotopteryx chenopodiata (L.) 134

Scythris 21

- acipenserella K. Nupponen \& T. Nupponen sp. n. 5, 7-9 
- aegrella K. Nupponen \& Junnilainen sp. n. 5, 9-10

- albisaxella K. Nupponen \& T. Nupponen sp. n. $10-11,15,17$

— arkaimensis Bengtsson sp. n. 5, 10, 11

- bengtssoni Patocka \& Liska 11

- bifissella (Hofmann) 11, 14

- braschiella (Hofmann) 11, 13

- brunneofasciella K. Nupponen \& Junnilainen sp. n. $5,7,11-12$

- bubaniae Walsingham 13

- clavella (Zeller) 12

- cretacella K. Nupponen \& T. Nupponen sp. n. 5, 12-13

- disparella (Tengström) 13

- elenae K. Nupponen sp. n. 8, 13

- emichi (Anker) 13

- ericetella (Heinemann) 13

- eversmanni K. Nupponen \& T. Nupponen sp. n. $5,8,14$

- flavilaterella (Fuchs) 15

- flaviventrella (Herrich-Schäffer) 15

- gozmanyi Passerin d'Entrèves 10, 15

- karinupponeni Bengtsson sp. n. 5, 15-16

- kasyi Hannemann 16

— kullbergi Bengtsson 11

- kyzylensis Bengtsson 17-18

— laminella (Denis \& Schiffermüller) 20

- luxatiella K. Nupponen \& Kaitila sp. n. 5, 1617

- mikkolai Sinev 15-17

- obscurella (Scopoli) 17

- olschwangi K. Nupponen \& T. Nupponen sp. n. $5,7,17-18$

- penicillata Chretien 17

- perlucidella K. Nupponen \& T. Nupponen sp. n. $5,7-8,18$

- picaepennis (Haworth) 11

- platypyga Staudinger 8

- productella (Zeller) 19

- pruinata Falkovitch 12

- pudorinella (Möschler) 19

— remexella K. Nupponen \& Kaitila sp. n 5, 7, 8, 19-20

- setiella (Zeller) 8, 20

— sinensis (Felder \& Rogenhofer) 20

- sitarcha Meyrick 8

- subaerariella (Stainton) 20

- sublaminella K. \& T. Nupponen sp. n. 5, 8, 20
- terekholensis Bengtsson 11

- tributella (Zeller) 21

Sedum telephium L. 114

Seleucus Holmgren 195-196, 201-202, 223

- cuneiformis Holmgren 195, 197, 201, 224 225

- exareolatus Strobl 201

Semioscopis steinkellneriana (Den. \& Schiff.) 133

Semiothisa clathrata (L.) 135

- liturata (Cl.) 135

Sigara 183

- distincta (Fieber) 184

- falleni (Fieber) 184

- fossarum (Leach) 184

- limitata (Fieber) 183, 184

— longipalis (J. Sahlberg) 183, 184

- semistriata (Fieber) 184

- striata (Linnaeus) 184

Siler cupreus 75

Sitticus 83

- catieri 83

— albolineatus (Kulczynski) 83

- avocator (=viduus) 70

— avocator (O.P.-Cambridge) 70, 83

- caricis (Westring) 70

- catieri 83

- cutleri Prószynski 83

- distinguendus (Logunov) 70

- distinguendus (Simon) 83

- eskovi Logunov \& Wesolowska 83

- fasciger(Simon) 83

- finschi (L. Koch) 83

- floricola 84

- lineolatus (Grube) 84

- penicillatus (Simon) 84

- pubescens (Fabricius) 70

- saxicola (C.L. Koch) 84

- terebratus (Clerck) 70

— viduus 70, 83-84

Sorbus sibirica Hedl. 142

Spaelotis lucens Btl. 154

Spatalia argentina D. \& S. 154

- sdoerriesi Graes. 154

Sphecodina Blanch. 153

Spilomena 35-36, 40-41, 46-48, 53-54

- beata Blüthgen $35-36,41-48,50,52-54$

- beatalexspectata 36

- beata-valkeilai 46 
- canariensis Blüthgen 41, 47-48

- curruca (Dahlbom) = S. pulawskii D 36

- curruca (Dahlbom), 35-41, 47-50, 53-54

- curruca (Lomholdt) 38

- curruca $=$ S. differens 49

- differens Blüthgen 35-41, 47-50, 52, 54

- enslini Blüthgen $35,37,41,46-47,48-50$, 54

- exspectata $41,43,46,52-53$

- exspectata Dollfuss 43

- exspectata Valkeila 35-36, 41, 52

- mocsaryi Kohl 41, 47-48, 54

- pulawskii Dollfuss 35-38, 47, 48, 54

- punctatissima Blüthgen 41, 47-48, 54

- troglodytes $37,41,46,48-49,52-54$

- troglodytes (= vagans) 49,53

- troglodytes (Vander Linden) 50

- troglodytes (Vander Linden) (= vagans Blüthgen) 47

- troglodytes-enslini 46

- vagans Blüthgen 41, 51-53

- valkeilai sp. n. 35-36, 41-47, 53-54

Spodoptera 185

Stenoptilia veronicae Karv. 134

Sterrhopterix standfussi (Wck.) 133

Stigmella myrtillella (Stt.) 131

Stipa 7

Stizostedion lucioperca 183

Strix aluco 98

—uralensis Pall. 98, 136

Stromatium fulvum (Villers) 2

Strophedra nitidana F. 154

Succisa pratensis 172

Swammerdamia compunctella (H.-S.) 133

Symphanes Foerster 239

- striolata (Thomson) 239, 242

Synageles hilarulus (C.L. Koch) 84

— morsei Logunov \& Marusik 84

- nigriculus Danilov 84

- venator (Lucas) 84

Synagelides agoriformis Strand in Bösenberg \& — zhilcovae Prószynski 84-85

\section{$\mathbf{T}$}

Taeniothrips 191

Talavera minuta (Banks) 85

Taleporia borealis (Wck.) 135

Telamonia castriesiana 80
Teleiodes notatella (Hb.) 134

Tenthredo 88

Tenthredopsis (Hinz) 88

Thersilochus minutus Bridgman 206

- nitidus Bridgman 204, 206

Thinodromus arcuatus (Stephens) 229

Thyatira batis (L.) 134

Tilia 142, 144, 196, 232, 233

- cordata L. 143

Tinea Zeller 98, 107, 136

- bothniella Svensson 107, 133

- columbariella Wocke 105, 107, 133

- pellionella 107

- sibiriella 133

- steueri Petersen 97-102, 105-107

- svenssoni Opheim 97-99, 102-107, 131, 133, 136

- trinotella Thnbg. 133

- unidentella 133

Tineola bisselliella 97

Trachyusa Ruthe 239

Trigona 194

Trombidium Fabricius 187, 190, 192

- holosericeum (Linnaeus) 187, 190, 192

Tsuga 144

$\mathbf{U}$

Ulmus 142, 154, 156, 232-234

- laevis Pall. 143

- pumila L. 143, 150

- japonica (Rehd.) Sarg.) 143

- macrocarpa Hance 143

Utetheisa Hbn. 153

V

Vaccinium uliginosum 135

— vitis-idaea L. 58

Valeriana sambucifolia 170

Vanessa atalanta (L.) 135

Vendolus Roman 215

- stilpninus Roman 215

Veronica chamaedrys 171

- longifolia 171

- spicata 169, 171

Viburnum opulus 171

Viola 114 
$\mathbf{X}$

Xanthia togata (Esp.) 56, 135

Xestia brunneopicta (Matsumura) 135-136

$\mathbf{Y}$

Yaginumaella 85

- medvedevi Prószynski 85
- striatipes (Grube) 85

Ypsolopha nemorella (L.) 133

- parenthesella (L.) 133

$\mathbf{Z}$

Zygaena 152 